\title{
Comparison of Anisotropic Analytical Algorithm and Acuros XB Calculation Algorithms on Intensity-modulated Radiotherapy and Volumetric Modulated Arc Therapy Techniques for Nasal Cavity and Paranasal Sinus Tumors: Effects on Integral Radiation Dose
}

\author{
(1) Aydın ÇAKIR, 1 (D) Züleyha AKGÜN² \\ 'İstanbul Bilgi University, Vocational School, İstanbul-Turkey \\ ${ }^{2}$ Department of Radiation Oncology, Memorial Şişli Hospital, İstanbul-Turkey
}

\begin{abstract}
OBJECTIVE
This study aims to investigate the dosimetric effects of Acuros XB (AXB) and Anisotropic Analytical algorithms (AAA) on intensity-modulated radiotherapy (IMRT) and volumetric modulated arc therapy (VMAT) techniques for nasal cavity and paranasal sinus tumors.

\section{METHODS}

This study included 10 patients with the nasal cavity and paranasal sinus tumors, and 7-field non-coplanar IMRT plan and VMAT plans were generated with 6-MV photon beams specially selected for each patient anatomy. The effects on planning target volume (PTV) and organ-at-risk (OAR) were evaluated using $\mathrm{AXB}$ and $\mathrm{AAA}$ in each treatment technique to compare the accuracy of the calculation.

\section{RESULTS}

Conformity Index (CI) values for PTV were found to be $1.02 \pm 0.02$ and $1.03 \pm 0.03$ for $\mathrm{VMAT}_{\mathrm{AAA}}$ and $\mathrm{VMAT}_{\mathrm{AXB}}$ plans, respectively and $1.18 \pm 0.03$ and $1.20 \pm 0.02$ for $\mathrm{IMRT}_{\mathrm{AAA}}$ and $\mathrm{IMRT}_{\mathrm{AXB}}$ plans, respectively. Regarding heterogeneity index (HI) values, $\mathrm{VMAT}_{\mathrm{AAA}}$ and $\mathrm{VMAT}_{\mathrm{AXB}}$ plans $(0.025 \pm 0.02 ; 0.029 \pm 0.02)$ were found to have better HI values than $\operatorname{IMRT}_{\mathrm{AAA}}$ and $\mathrm{IMRT}_{\mathrm{AXB}}$ plans $(0.246 \pm 0.02 ; 0.335 \pm 0.03)$. Depending on the technique and algorithm used, a dose difference of $4 \%-14 \%$ was detected between PTV Dmin values.
\end{abstract}

\section{CONCLUSION}

The selection of AXB algorithm in treatment regions with high tissue heterogeneity will give more accurate dose calculation results for PTV and healthy tissues.

Keywords: Anisotropic analytical algorithm; Acuros XB; nasal cavity, radiotherapy for paranasal sinus; integral dose. Copyright $\odot$ 2019, Turkish Society for Radiation Oncology

\section{Introduction}

Radiotherapy significantly contributes to reducing the risk of postoperative local recurrence in the treatment of nasal cavity tumors. The application of radiother- apy for nasal cavity tumors is very difficult due to the presence of critical organs and large air cavity in the treatment site. The build-up effect seen in the transition from air to tissue encounters a secondary build-up effect in the nasal region. It is very difficult to achieve 
homogenous dose distribution within the planned target volume (PTV). The surrounding tissues are exposed to high integral doses to remove the cold spots in the PTV.[1-3]

The integral dose (ID) is the volume integral of the dose stored in a medium and is equal to the average dose received by the medium multiplied by its volume. It is also the area under the differential absolute dosevolume histogram curve. Published studies suggest that a large number of beam and monitor units (MU) used in intensity-modulated radiotherapy (IMRT) may cause an increase in ID and high-energy photon beams substantially reduce ID. D'Souza et al. reported that the change in ID with four or more beams is a function of the number of beams. High-energy beams reduce the ID as expected. The reduction rate was reported to be $1.5 \%-1.7 \%$ for the nasopharynx, $0.9 \%-1.0 \%$ and $0.3 \%$ for the pancreas and $0.4 \%$ for the prostate. In different beam-weighted two, four, and eight-field plans, ID was reported as $1.4 \%-2.1 \%$ for the nasopharynx, $0.2 \%$ $1.3 \%$ for the pancreas and $0.5 \%$ for the prostate. These results show that the ID decreases with increasing tumor size for similar anatomical dimensions, whereas it increases with the increasing size of the anatomical region for similar tumor sizes.[4]

There are chemical structural elements in the human body, and it is, therefore, a medium with different density. Air, bone, adipose tissue and lungs measure about -1000 Hounsfield unit (HU), $+1000 \mathrm{HU},-50-100$ $\mathrm{HU}$ and $-500 \mathrm{HU}$, respectively. The reduction of radiation in the tissue is calculated with the help of computed tomography (CT) data and calibration curves using $\mathrm{HU}$ values obtained from $\mathrm{CT}$ and tables specific to predefined density ranges. The accuracy of the algorithms (dose calculation mechanisms) that can include tissue composition in determining the dose in each organ may be different.

In Eclipse ${ }^{\mathrm{Tm}}$ Treatment Planning System version 13.0 (Varian Medical Systems, Palo Alto, CA), the analytical anisotropic algorithm (AAA) method is widely used for the calculation of dose distributions.

There are studies in the literature reporting that the dose calculation made using AAA was significantly inaccurate. In particular, it has been observed that it calculates the dose inaccurate when near the two media during the transition from tissue to air.[5-6] Recently, a new dose calculation algorithm called Acuros XB (AXB) has been introduced by Varian (Varian Medical Systems, Palo Alto, CA) to fix this situation. Acuros $\mathrm{XB}$ uses a complex technique to solve Linear Boltzmann Transport Equation (LBTE) and provides an accurate approach to patient dose calculation with heterogeneities like air, lung, bone, and implants with different density. Linear Boltzmann Transport Equation describes the macroscopic behavior of the radiation beam in the medium through which it passes.[7-8]

To our knowledge, in the relevant literature, there are no studies emphasizing the importance of using calculation algorithms using VMAT and IMRT techniques for nasal cavity and paranasal sinus tumors. The most recent study on nasal cavity and paranasal sinus tumors was conducted by Jeong et al.[3] in 2014. They compared the dosimetric results of VMAT and IMRT techniques which were compared only regarding PTV and critical organ doses.

To contribute to the literature, the present study aims to investigate the effects of the calculation algorithm on treatment plans made using IMRT and VMAT techniques in radiotherapy of nasal cavity tumors. This study investigated the effects of calculation differences between AXB and AAA algorithms on PTV and critical organ doses for nasal and paranasal sinus tumors with large air mass.

\section{Materials and Methods}

\section{Eclipse Treatment Planning System}

Eclipse $^{\mathrm{Tm}}$ Treatment Planning System version 13.0 (Varian, Palo Alto, California, USA) is designed for three-dimensional conformal radiotherapy (3D CRT), IMRT, VMAT, stereotactic radiosurgery (SRS)/stereotactic body radiotherapy (SBRT) and electron planning. The Eclipse treatment planning system used in our clinic includes dose-volume optimizer (DVO), plan geometry optimization (PGO), progressive resolution optimizer (PRO), multi-resolution dose calculation (MRDC), pencil beam convolution (PBC), AAA and AXB algorithms.

\section{Analytical Anisotropic Algorithm}

The AAA dose calculation model is a three-dimensional (3D) pencil beam and convolution superposition algorithm consisting of separate models for primary photons, scattered photons, and electrons scattered from beam regulating devices (primary collimator, beam straightening filter, and wedge filter). The functional forms forming the basic physical quantities initiate a process by considering the device properties. This usually results in a significant reduction in the computational time required for such algorithms. Tissue heterogeneities are anisotropically taken into account in the 3-dimensional neighborhood using mul- 
tiple lateral photon scattering kernels. The final dose distribution occurs by overlapping the contribution of photon and electron beams. The AAA algorithm calculates the dose behind the airspace to some extent due to an error that arises from modelling the scattered dose.[9-11]

\section{Acuros XB Algorithm}

The AXB algorithm was developed for two strategic needs-accuracy and speed-in external photon beam treatment planning. Acuros XB uses a complex technique to solve Linear Boltzmann Transport Equation (LBTE) and fully exploits patient dose calculation for heterogeneities due to lung, bone, air and non-biological implants. [7]

Instead of Boltzman Transport Equation (BTE), which describes the macroscopic behaviour of radiation particles, LBTE - its linear form - assumes that interaction in the environment where radiation particles penetrate occurs without the particles contacting with each other in the medium and without an external magnetic field.[7-8] There are two solution approaches that try to explain LBTE. One of the approaches is the Monte Carlo method, which does not clearly solve LBTE and produces indirect solutions for LBTE. The second approach is solving LBTE using numerical methods.

Although Monte Carlo and LBTE solution methods provide similar results, they cannot produce clear solutions and result in errors. Monte Carlo errors are random and result from that a limited number of particles are simulated. Systematic errors may occur when the Monte Carlo method uses precise techniques to speed up solution time.

The source model of the AXB algorithm used in the Eclipse TPS uses the existing AAA source model. This model includes primary photons, out-of-focus photons, contaminant electrons and scattered photons.

Fogliata et al.[9] reported that a lower dose of 3\% to $6 \%$ was obtained with $\mathrm{AXB}$ on critical organs compared to AAA. They reported that a lower dose $(3.6 \%$ to $3.7 \%$ ) was obtained with $\mathrm{AXB}$ in the same volume of lungs receiving V 5 and $\mathrm{V} 20$ doses.

The AXB algorithm can calculate the dose more accurately than the AAA using the mass density information obtained from the CT images in each voxel for the dose calculation. The calculation difference between the two algorithms is affected by parameters, such as the energy of the incoming beam, the field size and the electron density of the medium.

\section{Treatment Planning of Nasal Cavity and Paranasal} Sinus Tumors

In this study, CT data with a $2 \mathrm{~mm}$ cross-sectional thickness of 10 patients with the nasal cavity and paranasal sinus tumors admitted to our clinic were used. Varian TrueBeam STx using 6 MV beams was used for treatment planning. Non-coplanar IMRT and VMAT plans were made through the Eclipse treatment planning system.

The selected dose calculation algorithm and techniques were compared. In the IMRT technique, model $\mathrm{IMRT}_{\mathrm{AAA}}$ was created for the AAA algorithm, and model IMRT $_{\text {AXB }}$ was created for the AXB algorithm. Similarly, in the VMAT technique, model VMAT AAA $_{\text {A }}$ was created for the AAA algorithm, and model VMAT $_{\mathrm{AXB}}$ was created for the AXB algorithm.

\section{a- IMRT Planning Technique}

For each patient's anatomy and tumor location, 7-field non-coplanar treatment areas were selected. Table angle was chosen as $90^{\circ}$ for non-coplanar areas in a way that the selected treatment areas were not parallel to each other. A collimator angle of $5-10^{\circ}$ was used to minimize the tongue-and-groove effect created by treatment areas.

\section{b- VMAT Planning Technique}

The beam angles were selected as follows: counterclockwise from $179.9^{0}-180.1^{0}$ with a collimator angle of $30^{\circ}$, a couch angle of $0^{\circ}$ and clockwise from $180.1^{\circ}$ $179.9^{\circ}$ with a collimator angle of $330^{\circ}$, a couch angle of $0^{\circ}$.

Treatment planning was performed for each patient using AAA and AXB algorithms. In all planning, the calculation grid size (CGS) of $1 \mathrm{~mm}$ was selected to reduce the effects of CGS on dose distribution.

\section{Dosimetric Evaluation of the Treatment Plans}

Each treatment plan was evaluated in terms of PTV and organ-at-risk (OAR) using dose-volume histograms (DVH) and taking into account the criteria of the Radiation Therapy Oncology Group (RTOG). In each treatment planning, 95\% of PTV was ensured to receive at least $50 \mathrm{~Gy}$ as the primary dose limitation. The followings were calculated: PTVD98, which was considered a low dose zone for PTV, PTV $\mathrm{D}_{2}$, which was a high dose zone for PTV, minimum dose of PTV $\left(\right.$ PTV $\mathrm{D}_{\text {min }}$ ), mean dose values received by PTV (PTV $\mathrm{D}_{\text {mean }}$ ), and heterogeneity index (HI) and conformality index (CI) for PTV. 


\section{Quality Assurance of the Treatment Plans}

Arc CHECK (Sun Nuclear Corporation, FL-USA) phantom providing 3D comparison was used for quality assurance (QA) of the patient treatment plans. Four different QA plans were prepared for each patient treatment plan using the IMRT and VMAT techniques. Dose difference (DD) and distance-to-agreement (DTA) were selected as $2 \%$ and $2 \mathrm{~mm}$ in gamma analysis.

\section{Results}

\section{a- Evaluation of Dose-Volume Histograms}

The dosimetric results for the treatment plans made using two different algorithms are shown in Table 1. Table 1 presents the $\mathrm{PTVD}_{98}$ that was considered a low dose zone for PTV, PTV $\mathrm{D}_{2}$ that was a high dose area for PTV, PTV $\mathrm{D}_{\text {min }}$, and PTV $\mathrm{D}_{\text {mean }}$. Furthermore, CI and $\mathrm{HI}$ values in PTV for both treatment techniques and calculation algorithms are shown. When the CI and $\mathrm{HI}$ values of the treatment plans were examined, $\mathrm{CI}$ and HI values were found to be higher in the treatment plans made using the VMAT technique compared to the IMRT technique. An example of a treatment plan calculated for two different algorithms using IMRT and VMAT treatment techniques is shown in Figure $1 \mathrm{a}-\mathrm{b}$, and comparative mean DVH for this study is shown in Figure 2 a-j.

When Table 1 is examined, it is seen that there is a significant difference between the two techniques concerning $\mathrm{HI}$ and CI values for PTV.
In terms of PTV $\mathrm{D}_{\text {min }}$ doses, the highest difference was observed between the IMRT $_{\mathrm{AAA}}$ and $\mathrm{VMAT}_{\mathrm{AXB}}$ plans, which was $14 \%$. The least difference was between the $\mathrm{IMRT}_{\mathrm{AAA}}$ and $\mathrm{VMAT}_{\mathrm{AAA}}$ plans, which was $4 \%$. This difference was due to the calculation algorithm, not the treatment technique used.

Concerning PTV $\mathrm{D}_{\text {mean }}$ doses, the highest difference was found to be between the IMRT $\mathrm{IAA}_{\text {aA }}$ and IMRT $_{\mathrm{AXB}}$ plans, which was $4 \%$, and the least difference was between the IMRT $_{\text {AAA }}$ and VMAT $_{\text {AAA }}$ plans, which was $<1 \%$.

Regarding PTV $\mathrm{D}_{2}$ doses, the highest difference was between the IMRT $_{\text {AAA }}$ and VMAT $_{\text {AXB }}$ plans, which was $6 \%$, and the least difference was between the IMRTAAA and VMAT ${ }_{\text {AAA }}$ plans, which was $<1 \%$.

In terms of PTV $D_{98}$ doses, the highest difference was between the IMRT $\mathrm{IAA}_{\text {And IMRT }}$ axB plans, which was $3 \%$, and the least difference was between the

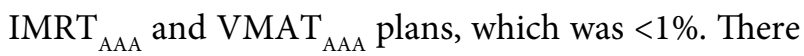
was a difference between $\mathrm{VMAT}_{\mathrm{AAA}}$ and $\mathrm{VMAT}_{\mathrm{AXB}}$.

When evaluated in terms of left and right optic nerve, there was a significant difference between IMRT and VMAT in all plans (IMRT ${ }_{\mathrm{AAA}}, \mathrm{IMRT}_{\mathrm{AXB}}$, VMAT $_{\text {AAA }}$, VMAT $_{\mathrm{AXB}}$ ).

When evaluated concerning optic chiasm doses, there was a significant difference between IMRT and VMAT in all plans IMRT $_{\text {AAA }}$, IMRT $_{\text {AXB }}$, VMAT $_{\text {AAA }}$, $\left.\operatorname{VMAT}_{\mathrm{AXB}}\right)$.

Concerning the left eye, there was a significant difference between $\mathrm{VMAT}_{\mathrm{AXB}}$ and $\mathrm{IMRT}_{\mathrm{AAA}}$ plans
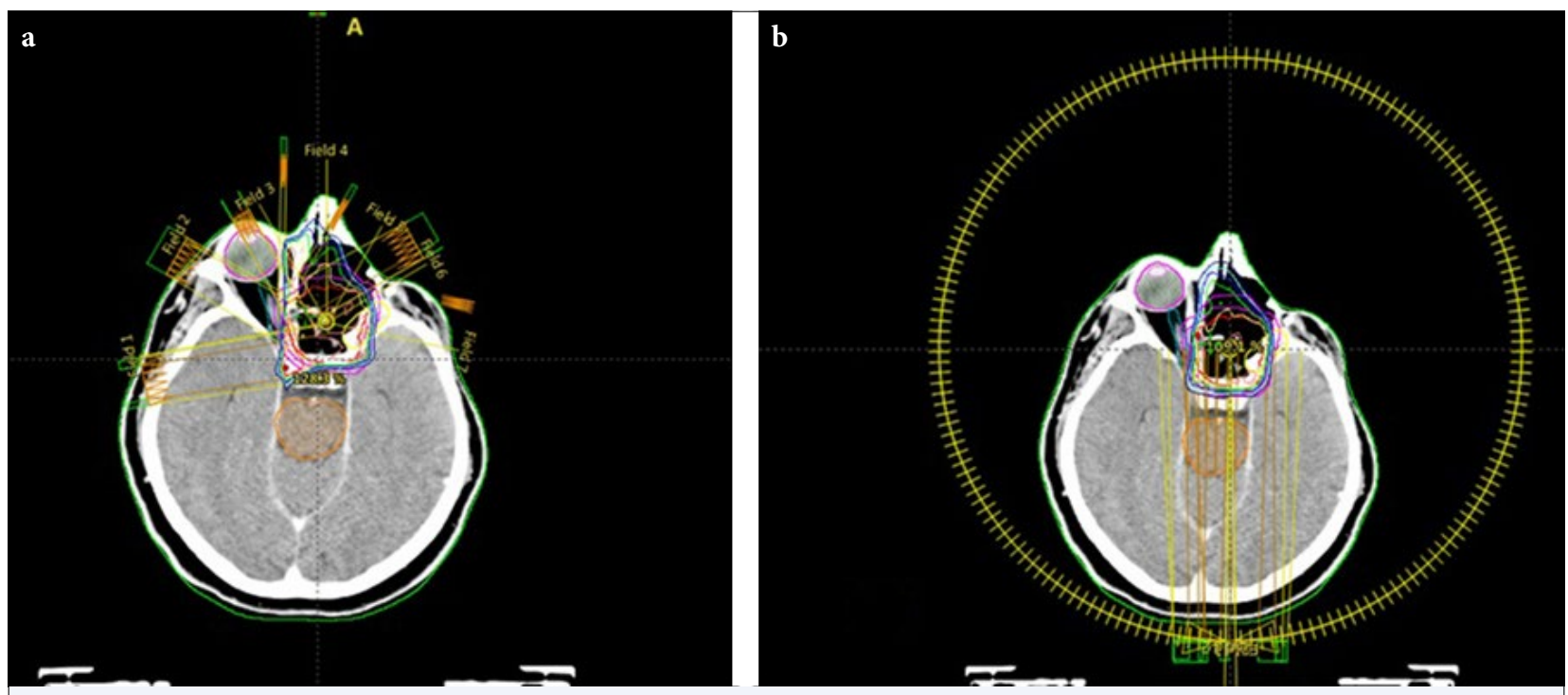

Fig. 1. (a) An example of the treatment plan for the IMRT technique. (b) An example of the treatment plan for VMAT technique. 


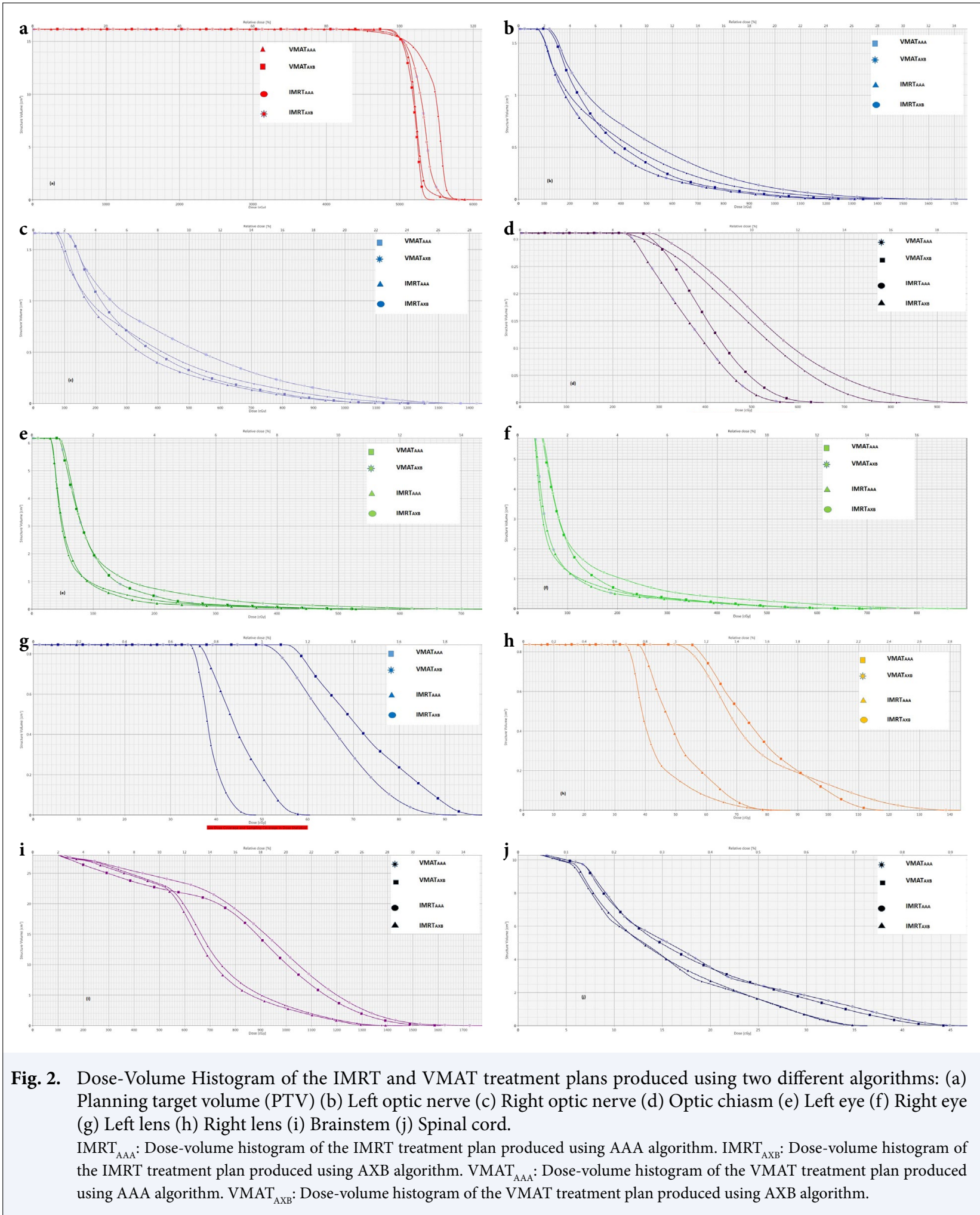

$(\mathrm{p}=0.027)$, whereas no significant difference was observed between $\mathrm{VMAT}_{\mathrm{AXB}}$ and $\mathrm{IMRT}_{\mathrm{AXB}}$ plans $(\mathrm{p}=0.062)$.
In terms of the right eye, there was a significant difference between VMAT $_{\text {AXB }}$ and IMRT $_{\text {AAA }}$ plans $(\mathrm{p}=0.039)$, whereas no significant difference was 
observed between VMAT $_{\mathrm{AXB}}$ and IMRT $_{\mathrm{AXB}}$ plans ( $\mathrm{p}=0.058)$.

Left and right lens doses were found to be higher in VMAT technique than in IMRT technique. In both techniques, the $\mathrm{AXB}$ algorithm determined a higher dose than AAA. This increase was due to the increase in small doses in the VMAT technique, which leads to some dose increase on critical organs with a small volume.

It can be further seen in Table 1 that there is some increase in the brainstem and spinal cord doses in the VMAT technique.

\section{b- Evaluation of the Quality Assurance of the Patient Treatment Plans}

Four different QA plans were prepared for each pa- tient treatment plan using the IMRT and VMAT techniques. Dose difference (DD) and distance-to-agreement (DTA) were selected as $2 \%$ and $2 \mathrm{~mm}$ in gamma analysis. Gamma analysis evaluations are shown in Table 2.

When Table 2 is examined, the choice of $\mathrm{AXB}$ instead of the AAA algorithm as the calculation algorithm in the IMRT and VMAT techniques had a significant effect on the results of gamma analysis evaluation. In both techniques, selecting the calculation algorithm as AXB increased the gamma passing rate to over $98 \%$. The AXB algorithm increased the consistency between the dose calculated on the treatment planning computer and the dose measured on the treatment device.

Table 1 Dosimetric results of treatment plans: IMRT ${ }_{A A A}$ : IMRT treatment plan produced using AAA algorithm. IMRT ${ }_{A X B}:$ IMRT treatment plan produced using the $A X B$ algorithm. VMAT ${ }_{A A A}: \mathrm{VMAT}$ treatment plan produced using AAA algorithm. $\mathrm{VMAT}_{\mathrm{AXB}}$ : Dosimetric results of VMAT treatment plan produced using AXB algorithm.

\begin{tabular}{|c|c|c|c|c|c|c|c|}
\hline \multirow[t]{2}{*}{ Parameter } & \multirow{2}{*}{$\begin{array}{c}\text { IMRT }_{\text {AAA }} \\
\text { Dose (cGy) } \\
\text { Mean士SD }\end{array}$} & \multirow{2}{*}{$\begin{array}{c}\text { IMRT }_{\text {AXB }} \\
\text { Dose (CGy) } \\
\text { Mean士SD }\end{array}$} & \multirow{2}{*}{$\begin{array}{c}\text { VMAT }_{\text {AAA }} \\
\text { Dose (cGy) } \\
\text { Mean士SD }\end{array}$} & \multirow{2}{*}{$\begin{array}{c}\text { VMAT }_{\text {AXB }} \\
\text { Dose (cGy) } \\
\text { Mean士SD }\end{array}$} & \multicolumn{3}{|c|}{$p\left(\right.$ VMAT $\left._{A X B}\right)$} \\
\hline & & & & & IMRT $_{\text {AAA }}$ & IMRT $_{\text {AXB }}$ & VMAT $_{\text {AAA }}$ \\
\hline PTV Dmin & $4549 \pm 137$ & $3931 \pm 110$ & $4344 \pm 423$ & $3948 \pm 269$ & 0.011 & 0.440 & 0.021 \\
\hline PTV Dmean & $5176 \pm 108$ & $5286 \pm 229$ & $5178 \pm 132$ & $5369 \pm 105$ & 0.035 & 0.048 & 0.022 \\
\hline PTV $D_{2}$ & $5298 \pm 148$ & $5479 \pm 298$ & $5332 \pm 121$ & $5609 \pm 28$ & 0.012 & 0.024 & 0.017 \\
\hline PTV D $_{98}$ & $4931 \pm 16$ & $4793 \pm 101$ & $4918 \pm 25$ & $4810 \pm 74$ & 0.033 & 0.067 & 0.032 \\
\hline $\mathrm{HI}$ & $0.335 \pm 0.03$ & $0.246 \pm 0.02$ & $0.025 \pm 0.02$ & $0.029 \pm 0.02$ & 0.012 & 0.032 & 0.025 \\
\hline $\mathrm{Cl}$ & $1.18 \pm 0.03$ & $1.20 \pm 0.025$ & $1.02 \pm 0.02$ & $1.03 \pm 0.03$ & 0.024 & 0.021 & 0.044 \\
\hline Left optic nerve & $3057 \pm 230$ & $3200 \pm 218$ & $2648 \pm 177$ & $3005 \pm 178$ & 0.048 & 0.034 & 0.001 \\
\hline Right optic nerve & $3113 \pm 258$ & $3216 \pm 251$ & $3098 \pm 267$ & $3351 \pm 270$ & 0.028 & 0.034 & 0.021 \\
\hline Optic chiasm & $2265 \pm 235$ & $2414 \pm 225$ & $2346 \pm 239$ & $2506 \pm 217$ & 0.018 & 0.038 & 0.032 \\
\hline Left eye & $1846 \pm 188$ & $1910 \pm 182$ & $1785 \pm 173$ & $2013 \pm 180$ & 0.027 & 0.062 & 0.018 \\
\hline Right eye & $2951 \pm 306$ & $2992 \pm 318$ & $2833 \pm 301$ & $2970 \pm 293$ & 0.039 & 0.058 & 0.033 \\
\hline Left lens & $306 \pm 150$ & $327 \pm 125$ & $560 \pm 153$ & $613 \pm 136$ & 0.002 & 0.001 & 0.045 \\
\hline Right lens & $325 \pm 162$ & $372 \pm 172$ & $553 \pm 161$ & $594 \pm 163$ & 0.001 & 0.002 & 0.048 \\
\hline Brainstem & $1633 \pm 210$ & $1650 \pm 150$ & $1717 \pm 148$ & $1710 \pm 189$ & 0.110 & 0.141 & 0.069 \\
\hline Spinal cord & $586 \pm 175$ & $593 \pm 178$ & $617 \pm 195$ & $624 \pm 181$ & 0.064 & 0.087 & 0.174 \\
\hline
\end{tabular}

Table 2 Gamma evaluation for treatment plans: IMRT ${ }_{A A A}$ IMRT treatment plan produced using the AAA algorithm. $I_{\text {IMRT }}$ : IMRT treatment plan produced using the AXB algorithm. VMAT ${ }_{A A A}: V_{M A T}$ treatment plan produced using AAA algorithm. VMAT $_{A X B}$ : Treatment plan quality control of the VMAT treatment plan produced using the AXB algorithm. The comparison of gamma index passing rates of the IMRT and VMAT planning at $2 \%$ dose difference (DD) and $2 \mathrm{~mm}$ distance to agreement (DTA) criteria.

\begin{tabular}{lcccccccccc} 
Patient number, (\%) & $\mathbf{1}$ & $\mathbf{2}$ & $\mathbf{3}$ & $\mathbf{4}$ & $\mathbf{5}$ & $\mathbf{6}$ & $\mathbf{7}$ & $\mathbf{8}$ & $\mathbf{9}$ & $\mathbf{1 0}$ \\
\hline $\mathrm{IMRT}_{\text {AAA }}$ & 97.6 & 96.4 & 98.2 & 97.8 & 97.2 & 98.2 & 98.4 & 97.4 & 98.2 & 98.1 \\
$\mathrm{IMRT}_{\text {AXB }}$ & 99.6 & 98.2 & 98.7 & 99.5 & 98.8 & 98.8 & 98.8 & 100 & 98.9 & 99.4 \\
VMAT $_{\text {AAA }}$ & 97.2 & 98.4 & 97.6 & 97.8 & 98.5 & 97.6 & 97.9 & 98.2 & 98.9 & 97.2 \\
VMAT $_{\text {AXB }}$ & 98.7 & 99.2 & 100 & 98.5 & 98.9 & 99.8 & 99.2 & 98.7 & 98.7 & 99.7 \\
\hline
\end{tabular}




\section{Discussion}

There are many studies on dosimetric phantom that aim to determine the calculation accuracy of treatment planning systems. Studies have shown that the AXB algorithm provides more accurate results than the AAA algorithm in heterogeneous environments. One of the factors affecting calculation accuracy is the selected CGS.[12-15] In our previous study, dose estimation has been shown to be correlated with CGS. Our study highlights a very important point that there is a $5 \%$ difference between AAA plans with 1-mm CGS and AXB plans with 1-mm CGS regarding PTV $\mathrm{D}_{\text {min }}$ values. There was a $4 \%$ difference between AAA plans with 1-mm CGS and AAA plans with 2.5-mm CGS, whereas there was a $1 \%$ difference between $\mathrm{AXB}$ plans with 1-mm CGS and AXB plans with 2.5-mm CGS. A significant improvement was observed in the dose accuracy of AXB plans with 1-mm CGS. The smaller CGS results in a better sampling of the structure voxels.[13]

In the phantom study with $6 \mathrm{MV}$ photon beams by Bush et al.,[6] they showed that there was a $4.5 \%$ difference between AXB and Monte Carlo algorithms in the transition from air to tissue, which increased to $13 \%$ with AAA algorithm. In parallel with this study, Kan et al.[16] reported in their dosimetric phantom study that there was a 3\% difference between the measurement and the calculated dose by AXB algorithm in the transition from air to tissue and this difference increased to $10 \%$ with AAA. In a phantom study by Suresh et al. on esophageal cancer, the dose of PTV $D_{\min }$ was calculated to be lower by $2.5 \%$ in AXB and by $9.1 \%$ in AAA.[17]

In our study that investigated the effects of the AAA algorithm and AXB algorithm on critical organ doses in breast radiotherapy, the findings showed that AAA calculated $2 \%, 2 \%, 8 \%$, and $4 \%$ more dose for the left lung, heart, contralateral breast, and contralateral lung, respectively.[18]

In a study conducted by Padmanaban et al.,[19] $\mathrm{AAA}$ and $\mathrm{AXB}$ algorithms were compared using 3D conformal, and VMAT techniques in the treatment of esophageal cancer and the AXB algorithm was found to determine a low dose in PTV (0.5-1.3 Gy) compared to AAA. They showed that the low dose in PTV obtained for AXB was not related to the technique used.

The most remarkable side of our study was that the dose of PTV, which started after the air cavity, was calculated higher with the AAA algorithm. There was a $14 \%$ between AAA plans and AXB plans in determining PTV $\mathrm{D}_{\text {min }}$ dose. A higher dose than should be in the build-up area between air and tissue was obtained with the AAA algorithm. The higher dose in PTV will increase the maximum dose effect in the hot dose regions as a result of the normalization of the plan to the treatment dose.

The literature review has shown that there are no studies emphasizing the importance of using calculation algorithms with the VMAT and IMRT techniques for nasal cavity and paranasal sinus tumors. The most recent study on nasal cavity and paranasal sinus tumors was conducted by Jeong et al. in 2014 in which the dosimetric results of the VMAT and IMRT techniques were compared. In this study, the IMRT and VMAT treatment techniques were compared only in terms of PTV and critical organ doses. [3]

Compared to the IMRT technique, the VMAT technique provides great convenience concerning optimization. Variable gantry speeds, simultaneous multi-leaf collimator (MLC) motion, and dose rate variability allow the dose to be adjusted at the desired site. However, the VMAT technique shows an increase in some low dose sites compared to the IMRT technique. This increase leads to an increase in critical organ doses, particularly in healthy tissues with a small volume.[20]

\section{Conclusion}

The present study has revealed that the calculation differences between AXB and AAA algorithms used in the radiation therapy for nasal cavity and paranasal sinus cancers caused significant differences in the stored integral doses on critical organs. The AAA algorithm calculates the dose behind the airspace to some extent due to an error resulting from modelling the scattered dose.

In daily patient set-ups, two-dimensional (2D) image registration using $\mathrm{kV}-\mathrm{kV} / \mathrm{MV}-\mathrm{kV}$ or imageguided radiation therapy (IGRT) methods like three-dimensional cone beam computerized tomography (CBCT) allow the correction of changes to occur in patient anatomy. The applicability of non-coplanar IMRT plans is more difficult than the VMAT technique. There is no possibility of image acquisition for each treatment area and table angle. Taking these difficulties into consideration, the VMAT technique will be more appropriate for both patient positioning and treatment.

In conclusion, radiotherapy for nasal cavity tumors and the accuracy of dose delivery are quite difficult due to the anatomical structure of the region, where we are pushing critical dose limits for critical organs, and 
different density tissues. Similar to the different tissue densities within the treatment area, many devices increasing the dosimetric uncertainty due to the patient stabilizing devices also affect the dose in the patient. It should be noted that treatment planning algorithms do not have the ability to accurately calculate the dose during air-to-tissue transitions. The AAA algorithm calculates the dose behind the airspace to some extent due to an error resulting from modelling the scattered dose. It should be further kept in mind that the VMAT technique provides similar and even better results with the IMRT technique regarding $\mathrm{HI}$ and $\mathrm{CI}$ evaluation. The selection of the AXB algorithm in the VMAT technique is of great importance for the accuracy of the calculation and for evaluating the doses to be received by the critical organs.

Peer-review: Externally peer-reviewed.

Conflict of Interest: None declared.

Financial Support: None declared.

Authorship contributions: Concept - A.Ç., Z.A..; Design - A.Ç., Z.A.; Supervision - A.Ç., Z.A.; Materials - A.Ç., Z.A.; Data collection \&/or processing - A.Ç., Z.A.; Analysis and/ or interpretation - A.Ç., Z.A.; Literature search - A.Ç., Z.A.; Writing - A.Ç., Z.A.; Critical review - A.Ç., Z.A.

\section{References}

1. Huang D, Xia P, Akazawa P, Akazawa C, Quivey JM, Verhey LJ, et al. Comparison of treatment plans using intensity-modulated radiotherapy and three-dimensional conformal radiotherapy for paranasal sinus carcinoma. Int J Radiat Oncol Biol Phys 2003;56(1):158-68.

2. Sheng K, Molloy JA, Larner JM, Read PW. A dosimetric comparison of non-coplanar IMRT versus Helical Tomotherapy for nasal cavity and paranasal sinus cancer. Radiother Oncol 2007;82(2):174-8.

3. Jeong Y, Lee SW, Kwak J, Cho I, Yoon SM, Kim JH, et al. A dosimetric comparison of volumetric modulated arc therapy (VMAT) and non-coplanar intensity modulated radiotherapy (IMRT) for nasal cavity and paranasal sinus cancer. Radiat Oncol 2014;9:193.

4. D'Souza WD, Rosen II. Nontumor integral dose variation in conventional radiotherapy treatment planning. Med Phys 2003;30(8):2065-71.

5. Vassiliev O, Wareing T, McGhee J, Failla G, Salehpour M, Mourtada F. Validation of a New Grid Based Blotzmann Equation Solver for Dose Calculation in Radiotherapy with Photon Beams. Phys Med Biol 2010;55(3):581-98.
6. Bush K, Gagne IM, Zavgorodni, Ansbacher S, Beckham W. Dosimetric Validation of Acuros XB with Monte Carlo Methods for Photon Dose Calculations. Med Phys 2011;38(4):2208-21.

7. Hoffmann L, Jørgensen MB, Muren LP, Petersen JB. Clinical Validation of the Acuros XB Photon Dose Calculation Algorithm, a Grid-Based Boltzmann Equation Solver. Acta Oncol 2012;51(3):376-85.

8. Han T, Mikell J, Salehpour M, Mourtada F. Dosimetric Comparison of Acuros XB Deterministic Radiation Transport Method with Monte Carlo and Model Based Convolution Methods in Heterogeneous Media. Med Phys 2011;38(5):2651-64.

9. Fogliata A, Nicolini G, Clivio A, Vanetti E, Cozzi L. Critical appraisal of Acuros XB and Anisotropic Analytic Algorithm dose calculation in advanced nonsmall-cell lung cancer treatments. Journ Clin Oncol 2012;83(5):1587-95.

10. Fogliata A, Nicolini G, Clivio A, Vanetti E, Cozzi L. On the dosimetric impact of inhomogeneity management in the Acuros XB algorithm for breast treatment. Radiat Oncol 2011;6(1):103.

11. Han T, Mourtada F, Kisling K, Mikell J, Followill D, Howell R. Experimental Validation of Deterministic Acuros XB Algorithm for IMRT and VMAT Dose Calculation with Radiological Physics Center's Head and Neck Phantom. Med Phys 2012;39(4):2193-2202.

12. Huang B, Wu L, Lin P, Chen C. Dose calculation of Acuros XB and Anisotropic Analytical Algorithm in lung stereotactic body radiotherapy treatment with flattening filter free beams and the potential role of calculation grid size. Radiat Oncol 2015;10:53.

13. Cakir A. Dosimetric comparison of Anisotropic Analytical Algorithm and Acuros XB in Stereotactic Body Radiotherapy and effect of calculation grid size. Turk J Oncol 2017;32(3):100-5.

14. Chung H, Jin H, Palta J, Suh TS, Kim S. Dose variations with varying calculation grid size in head and neck IMRT. Phys Med Biol 2006;51(19):4841-56.

15. Mittauer K, Lu B, Yan G, Kahler D, Gopal A, Amdur $\mathrm{R}$, et al. A study of IMRT planning parameters on planning efficiency, delivery efficiency, and plan quality. Med Phys 2013;40(6):061704.

16. Kan M, Leung L, Yu P. Verification and Dosimetric Impact of Acuros XB Algorithm on Intensity Modulated Stereotactic Radiotherapy for Locally Persistent Nasopharyngeal Carcinoma. Med Phys 2012; 39(8):4705-14.

17. Suresh R, Kevin R, Shyam P, Terry L, Daniel R, Christopher B. Acuros XB Algorithm vs. Anisotropic Analytical Algorithm: A Dosimetric study using hetergeneous phantom and computed tomography (CT) data sets of esophageal cancer patients. Journal of Cancer Therapy 2013;4(1):138-44. 
18. Cakir A, Akgun Z. Dosimetric comparison of integral radiation dose: Anisotropic Analytical Algorithm and Acuros XB in Breast Radiotherapy. Int Journ of Medical Phys, Clin Eng and Radiat Oncol 2019;8:57-67.

19. Padmanaban S, Warren S, Walsh A, Partridge M, Hawkins A. Comparison of Acuros (AXB) and Anisotropic Analytical Algorithm (AAA) for dose cal- culation in treatment of oesophageal cancer: effects on modelling tumour control probability. Radiat Oncol 2014;9:286.

20. Guckenberger M, Richter A, Krieger T, Wilbert J, Baier $\mathrm{K}$, Flentje $\mathrm{M}$. Is a single arc sufficient in volumetricmodulated arc therapy (VMAT) for complex-shaped target volumes? Radiother Oncol 2009; 93(2):259-65. 